\title{
Drought Responses in Six Hazelnut (Corylus avellana L.) Cultivars
}

\author{
Ali SHAHI-GHARAHLAR ${ }^{1,2}$, Mohammad Reza FATAHI ${ }^{1}$, Zabihollah ZAMANI ${ }^{1}$, Mohamad AL HASSAN ${ }^{2}$, \\ Monica BOSCAIU ${ }^{3 *}$, Oscar VICENTE ${ }^{2}$ \\ ${ }^{1}$ Department of Horticultural Sciences, University of Tehran, Iran \\ ${ }^{2}$ Instituto de Biología Molecular y Celular de Plantas (UPV-CSIC) and ${ }^{3}$ Instituto Agroforestal \\ Mediterráneo (UPV), Universitat Politècnica de València, Spain. \\ *)Corresponding author, e-mail: mobosnea@eaf.upv.es
}

BulletinUASVM Horticulture 73(2) / 2016

Print ISSN 1843-5254, Electronic ISSN 1843-5394

DOI:10.15835/buasvmcn-hort:12288

\begin{abstract}
Drought is one of the major causes of reduction of crop yields worldwide, a problem that will increase in the next decades due to climate change. We describe here an initial attempt to define biochemical markers associated to water stress in several hazelnut cultivars, by measuring the levels of common osmolytes and the generation of secondary oxidative stress, in plants subjected to water stress, and after recovery from the stress treatment. Proline (Pro) appears to be a reliable marker in this species, as its accumulation in leaves correlates well with the degree of stress affecting the plants. Differences between cultivars in relative Pro accumulation and oxidative stress suggested that some cultivars are more tolerant than others and could be selected for cultivation in droughtaffected areas.
\end{abstract}

Keywords: climate change; Corylus avellana; osmolytes; oxidative stress; water stress

\section{Introduction}

Drought is probably the environmental factor causing the most important reductions of crop yields worldwide; this problem will worsen in the near future due to the forecasted effects of climate change, especially in arid and semi-arid regions (Fita et al., 2015). Therefore, there is an obvious interest in investigating the mechanisms of drought tolerance in different crop species. The possibility of finding appropriate conditions for recovering water-stressed plants, by watering them before drought becomes lethal, has attracted less attention, but may help to establish more efficient crop management practices in a scenario of increasing scarcity of water resources.

\section{Aims and objectives}

This study represents an initial attempt to identify some biochemical markers that could be associated to the activation and inactivation of specific responses to water stress, in different Corylus avellana cultivars. These responses to drought include the accumulation of specific osmolytes and the generation of oxidative stress.

\section{Materials and methods}

A water-stress treatment (by completely stopping watering) was applied to three-year-old trees of six different $C$. avellana cultivars during ten days; then the trees were watered again. Nonstressed plants were maintained in parallel, as controls. Leaves were collected from the trees at the end of the drought treatment and after 24 hours of recovery. This material was used to determine the levels of some common osmolytes [proline (Pro) and total soluble sugars (TSS)], as well as malondialdehyde (MDA, an oxidative stress biomarker) contents, by spectrophotometric methods.

\section{Results and discussion}

After the drought treatment, measured Pro contents were higher than in the corresponding control plants, in all tested hazelnut cultivars, 

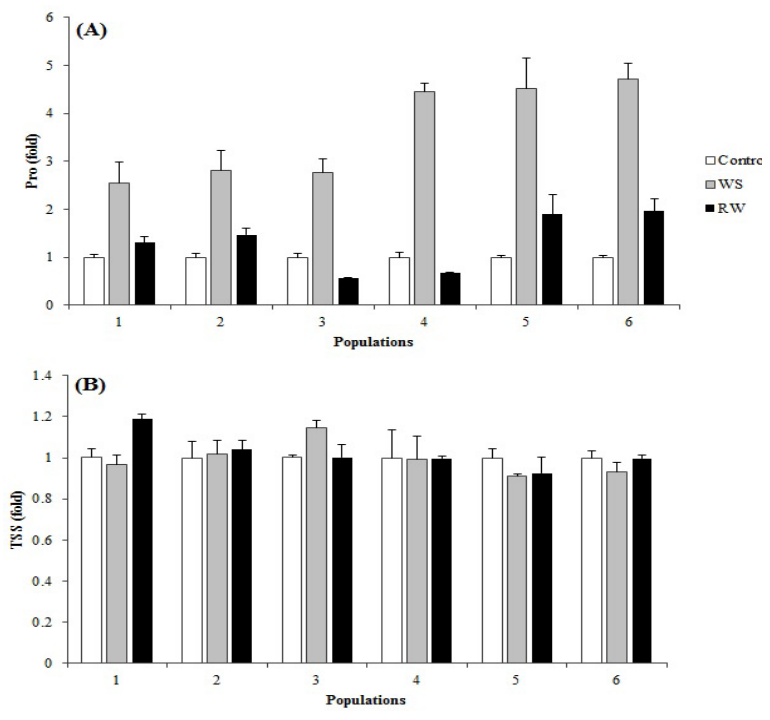

Fig. 1. (A) Proline (Pro) and (B) total soluble sugars (TSS) leaf levels in six populations (six cultivars of different origins) of Corylus avellana, in control, non-stressed plants (control), after 10 days of water stress treatment (WS) and after $24 \mathrm{~h}$ of recovery by re-watering the water-stressed plants (RW)

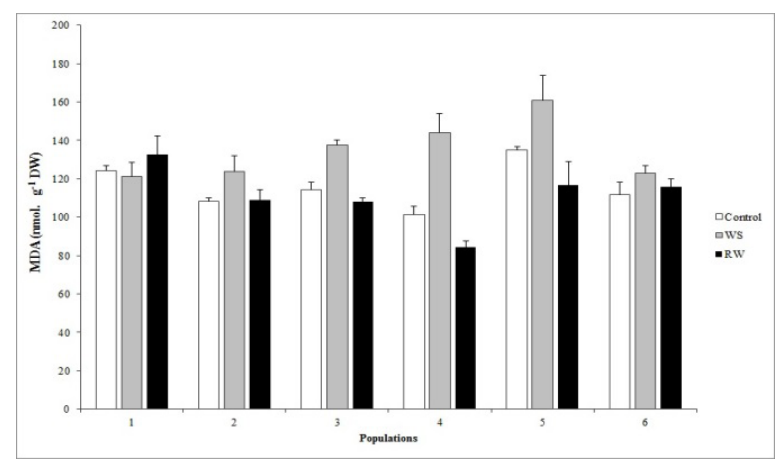

Fig. 2. Malondialdehyde (MDA) leaf levels in the six populations of Corylus avellana, in control, non-stressed plants (control), after 10 days of water stress treatment (WS) and after $24 \mathrm{~h}$ of recovery by re-watering the water-stressed plants (RW)

although the relative increases varied in different cultivars, between ca. 2.5-fold and almost 5-fold. One-day recovery, was sufficient to decrease Pro levels to values similar to those of the nonstressed controls (Fig. 1A). Therefore, there is a good correlation between Pro accumulation and the level of stress affecting the hazelnut plants.

No significant differences in TSS levels were detected between treatments (control, water stress, and water stress + recovery), for any of the six cultivars (Fig. 1B). This means that soluble carbohydrates do not accumulate in response to drought and probably have no functional role as compatible solutes in the mechanisms of stress response in this species.
In general, in most cultivars, MDA levels increased in response to water stress and decreased again after re-watering the trees (Fig. 2.). Yet the differences between treatments were significant only in some cultivars, which were more affected by oxidative stress.

\section{Conclusion}

Proline can be considered as a reliable marker of water stress in hazelnut. The differences observed between cultivars in the relative stressinduced proline accumulation and in the degree of oxidative stress, suggested that some cultivars are more tolerant to water stress than others and, 
after further characterisation, could be selected for cultivation in drought-affected areas.

\section{REFERENCES}

1. Fita, A., Rodríguez-Burruezo, A., Boscaiu, M., Prohens, J. and Vicente, O. (2015). Breeding and domesticating crops adapted to drought and salinity: A new paradigm for increasing food production. Front Plant Sci 6:978. doi: 10.3389/fpls.2015.00978. 